\title{
L'impegno di AIAF Onlus per le persone con malattia di Anderson-Fabry
}

Giornale di Tecniche Nefrologiche e Dialitich 20I8, Vol. 30(3-4) 230-233

(C) The Author(s) 2019

Article reuse guidelines:

sagepub.com/journals-permissions

DOI: $10.1177 / 0394936219832319$

journals.sagepub.com/home/gtn

(S)AGE

\author{
Stefania Tobaldini
}

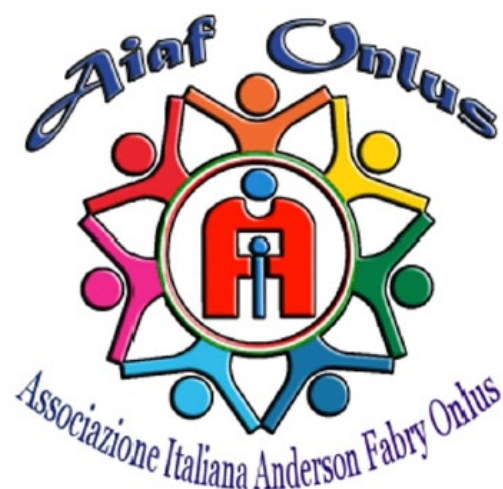

Aiaf Onlus (Associazione Italiana Anderson-Fabry) ̀̀ l'associazione di riferimento per i pazienti italiani con malattia di Anderson-Fabry. Svolgiamo sostegno e forniamo sevizi ai pazienti e alle loro famiglie attuando anche azioni di lobbying su obiettivi specifici che interessano il presente e il futuro di quanti in Italia vivono quotidianamente la malattia.

\section{Le attività}

Nel corso del 2018 abbiamo finanziato ed avviato il "Progetto di Dosaggio Lyso Gb-3", nato dalla volontà dell'associazione di dedicare un assegno di ricerca in onore del Dott. Gino Di Nella (fondatore del Gruppo Italiano Pazienti Fabry, ora confluito in Aiaf Onlus), che è venuto a mancare nel 2015.

Lo studio, in questa prima parte del progetto, coinvolgerà 100 pazienti italiani con malattia di Fabry di età compresa tra i 4 e i 75 anni (afferenti a 11 centri italiani di riferimento per diagnosi e follow-up). I pazienti saranno sottoposti ad un prelievo di campione di sangue intero e di alcune gocce di sangue raccolto su cartoncino (DBS) per stabilire una correlazione tra i valori riscontrati. Il progetto è stato redatto con il supporto del Comitato Scientifico di Aiaf in collaborazione con il Prof. Giancarlo La Marca, direttore del Dipartimento di Scienze biomediche, sperimentali e cliniche dell'Università di Firenze. Lo studio, approvato dal Comitato Etico Regionale della Toscana nel settembre 2017, si propone di dimostrare che il Lyso-Gb3 è in grado di diagnosticare la Malattia di
Fabry, verificando anche se il marcatore può dare indicazioni sull'efficacia delle terapie somministrate ai pazienti. L'auspicio di Aiaf è che i risultati confermino la validità di questa metodica, nell'ottica di dare un seguito a questo progetto e mettere a disposizione di tutti pazienti italiani il dosaggio di questo marcatore e, successivamente, proporre l'inserimento di questo dosaggio tra gli esami garantiti dal Servizio Sanitario Nazionale.

Verso la fine del 2018, abbiamo inoltre lanciato l'Indagine Nazionale sulla qualità della vita dei pazienti Anderson-Fabry, che ha visto un'ampia partecipazione da parte dei pazienti, i cui risultati saranno presentati nel corso del Meeting Nazionale Pazienti 2019 (che si terrà il 22 e 23 marzo). Si tratta di un progetto che riempie una lacuna: non esistono infatti ad oggi dati precisi e aggiornati sul numero di persone affette dalla patologia, ma, soprattutto, non esiste una seria valutazione dei costi diretti, indiretti e della qualità della vita dei pazienti.

Stiamo inoltre mettendo a punto un nuovo progetto, che abbiamo denominato "Caring Fabry", che si propone l'obiettivo di dare forma ad un Modello Ideale di Presa in Carico operativo della persona con Anderson-Fabry, da "esportare" poi in tutti i Centri di riferimento italiani.

Molti, infine, gli eventi organizzati, come il Meeting Nazionale dei pazienti, gli Incontri Regionali medicipazienti in Lombardia ed Emilia-Romagna, nonché i convegni a cui Aiaf ha partecipato.

\section{Le malattie lisosomiali nello screening neonatale esteso}

Il 2018 è stato un anno molto significativo per Aiaf, impegnata insieme ad altre Associazioni in iniziative

Presidente Aiaf Onlus - Associazione Italiana Anderson-Fabry, Italy

Corresponding author:

Stefania Tobaldini, Presidente Aiaf Onlus - Associazione Italiana

Anderson-Fabry, Italy.

E-mail: presidente@aiaf-onlus.org 
strategiche su due obiettivi molto importanti e concreti: l'estensione della terapia domiciliare (per i pazienti in terapia enzimatica sostitutiva) in tutte le Regioni italiane e l'inclusione delle malattie lisosomiali nello screening neonatale esteso.

Per quanto riguarda quest'ultimo, il risultato è stato raggiunto: nel novembre scorso, abbiamo promosso e sottoscritto insieme ad altre 15 Associazioni e Fondazioni un appello pubblico per appoggiare un emendamento alla Legge di Bilancio che prevedeva l'inserimento nello screening neonatale esteso (L.167/2016) anche delle malattie neuromuscolari genetiche, le immunodeficienze congenite severe e delle malattie da accumulo lisosomiale. Grazie alla nostra pressione, con l'approvazione della nuova Legge di Stabilità dal $1^{\circ}$ gennaio 2019 la Legge $167 / 2016$ è stata modificata con il testo dell'emendamento. Una svolta epocale per i pazienti con malattia di AndersonFabry, che potranno essere identificati già al momento della nascita e quindi godere dei vantaggi di una diagnosi precoce. Certo, la strada da fare per arrivare al concreto allargamento dello screening neonatale è ancora lunga e impegnativa e vedrà coinvolti molti attori: il Ministero della Salute, l'Istituto Superiore di Sanità, Age.na.s., Regioni e Province autonome di Trento e di Bolzano e le Società Scientifiche di settore. Ma Aiaf ci sarà e sarà pronta a collaborare per quanto necessario.

\section{L'impegno per ottenere la terapia enzimatica sostitutiva domiciliare in tutte le Regioni italiane}

Sul fronte della terapia domiciliare, il tema è stato affrontato più volte da Aiaf in occasione di convegni cui l'associazione è stata invitata. Azioni di sensibilizzazione che hanno trovato il loro culmine il 24 ottobre scorso con il convegno "Home Therapy per una migliore qualità di vita”. Tale convegno, realizzato in un'aula del Senato della Repubblica con il supporto dell'Intergruppo Parlamentare per le Malattie Rare nella persona della sua Presidente Senatrice Paola Binetti, è stato organizzato grazie ad un lavoro in sinergia durato due anni, da AIAF insieme ad AIG (Associazione Italiana Gaucher Onlus), AIG (Associazione Italiana Glicogenosi Onlus) e AIMPS (Associazione Italiana Mucopolisaccaridosi Onlus) (Fig. 1).

Un'occasione importante per sottoporre ai decisori, e in particolare alle Regioni che ancora non si sono attivate, ovvero Piemonte, Trentino, Emilia-Romagna, Marche, Umbria e Toscana, il tema della terapia domiciliare, anche portando l'esperienza di alcuni pazienti e l'umanità delle loro storie.

Nel corso del Convegno le Associazioni, insieme ad istituzioni, clinici e tecnici hanno affrontato il tema dell'importanza di garantire su tutto il territorio nazionale la terapia enzimatica sostitutiva (Ert) a domicilio, per evitare ai pazienti continui accessi in ospedale.

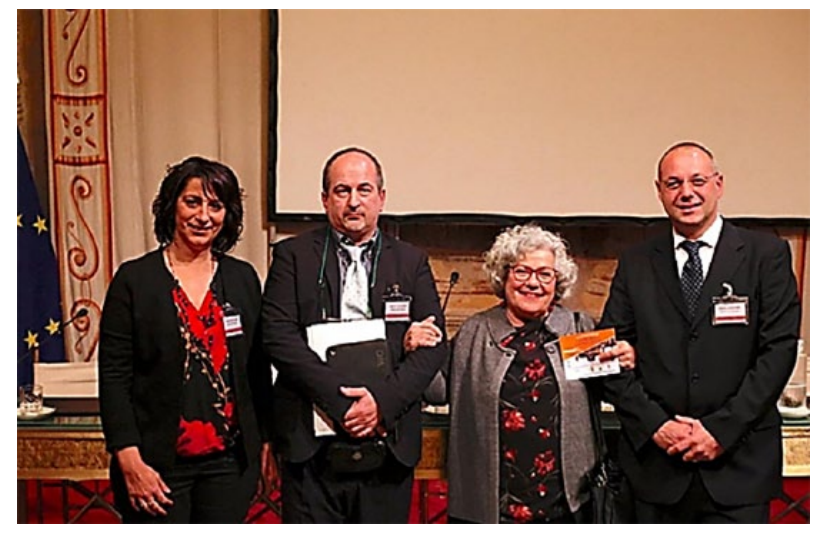

Fig I. I Presidenti delle quattro Associazioni Pazienti Malattie Lisosomiali.

Attualmente ogni Regione si comporta in modo differente nonostante l'accesso alla terapia domiciliare sia stato previsto dal "Documento sulla somministrazione a domicilio di farmaci ad alto costo per persone con malattie rare" del 2012, approvato dalla Commissione Salute e dalla Conferenza Regioni PPAA e presentato a Farmindustria: "Per alcuni prodotti farmaceutici spesso ad alto ed altissimo costo, specialmente per le malattie rare, la possibilità di somministrazione domiciliare è prevista dai decreti autorizzativi alla messa in commercio - si legge nel documento - L'accesso a tale somministrazione è un diritto del paziente, qualora le sue condizioni cliniche lo consentano. L'offerta dell'assistenza domiciliare, anche per quanto riguarda queste somministrazioni, attraverso il'SSR è compito che tutte le Regioni e le Province Autonome devono assolvere. Tale offerta (...) deve essere garantita dal servizio pubblico, a ciò deputato, anche se può in taluni casi essere integrata da un'offerta privata, secondo le procedure di acquisizione di servizi che ogni Regione o Provincia Autonoma ha definito".

A seguito di questo documento, alcune Regioni italiane si sono attivate, adottando specifiche delibere o determine, mentre altre hanno recepito le indicazioni tacitamente. Purtroppo non tutte. Nel caso della malattia di Fabry, malattia di Gaucher e Mucopolisaccaridosi, l'Ert in regime domiciliare è possibile solo in alcune Regioni: ben organizzati sono Lazio e Veneto insieme al Sud Italia e alle Isole. Le Marche hanno avviato da marzo la sperimentazione, mentre ancora non la concedono Piemonte, Trentino, Emilia-Romagna, Umbria e Toscana. Per la Glicogenosi, invece, la terapia domiciliare non è consentita in nessuna Regione, nonostante in altri Paesi europei sia già attiva.

La terapia domiciliare può essere fornita anche grazie alla collaborazione delle aziende farmaceutiche, che sostengono direttamente i costi della somministrazione, la quale può essere affidata ad aziende private specializzate in convenzione con le strutture pubbliche, laddove queste non riescano ad avviarla. 


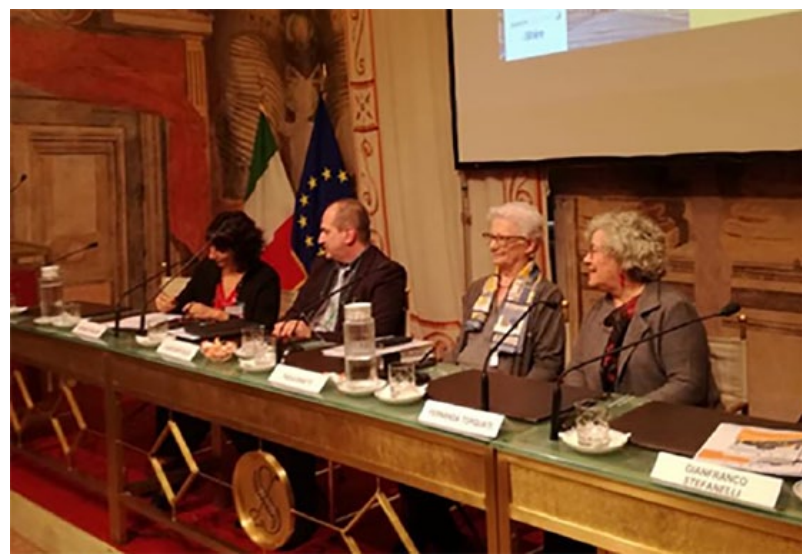

Fig 2. L'atto della firma del Patto d'Intesa tra Associazioni Pazienti e Istituzioni.

È stata evidenziata l'estrema convenienza della terapia domiciliare per i bilanci sanitari regionali, che, in base alle relazioni presentate al convegno, consentirebbe di risparmiare al Servizio pubblico le spese per i day hospital, valutate tra i 250 e i 500 euro al giorno a paziente, con risparmi che la Regione Veneto ha stimato intorno al $70 \%$ rispetto alla terapia in ospedale. Infine, si è ribadita la necessità che si provveda a un Piano nazionale, regole, protocolli e linee guida condivise e uniformi per tutto il territorio italiano.

Al termine della giornata, le Associazioni hanno proposto alle Istituzioni un Patto d'Intesa (Fig. 2) in cui sono stati delineati i punti fondamentali per estendere la terapia domiciliare in tutte le Regioni. Il Patto è stato firmato dalla Senatrice Binetti che ha assicurato che riferirà all'Intergruppo Parlamentare i temi emersi (Fig. 3).

\section{$E$ in futuro?}

Nei prossimi mesi, l'impegno di Aiaf proseguirà a fianco delle altre Associazioni con attività di sensibilizzazione e iniziative a livello regionale che coinvolgeranno i pazienti.

Tra queste, una raccolta di richieste di attivazione di terapia domiciliare da parte dei pazienti in cura con terapia enzimatica sostitutiva residenti nelle Regioni dove questa opzione non viene ancora consentita. I moduli compilati e sottoscritti congiuntamente dai pazienti e dai

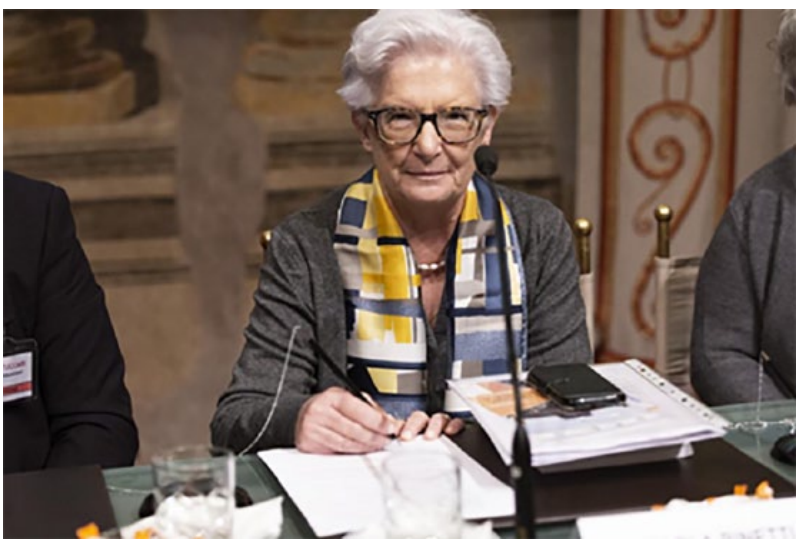

Fig 3. La Senatrice Paola Binetti, Presidente dell'Intergruppo Parlamentare per le Malattie Rare.

medici di riferimento (questi ultimi chiamati a confermare l'eleggibilità del loro paziente al trattamento domiciliare), saranno raccolti da ciascuna delle quattro associazioni ognuna per la propria patologia e verranno consegnati tutti insieme ai referenti della Sanità delle diverse Regioni.

In questo percorso sarà fondamentale l'unione delle forze di Associazioni, pazienti e clinici di riferimento per il raggiungimento di un obiettivo così importante come l'equità di trattamento per tutti i pazienti italiani. Una nuova ed impegnativa sfida che Aiaf vuole portare avanti.

Per richiedere il modulo scrivere a presidente@ aiaf-onlus.org

Ringrazio la Rivista "Giornale di Tecniche Nefrologiche e Dialitiche" per la preziosa opportunità di mettere in risalto un tema così importante per $i$ pazienti in cura con terapia enzimatica sostitutiva.

Stefania Tobaldini

Presidente Aiaf Onlus www.aiaf-onlus.org

E-mail: presidente@aiaf-onlus.org

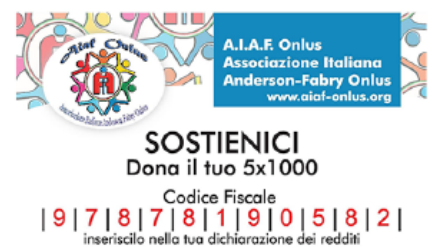


I vantaggi della terapia domiciliare per i pazienti con malattia lisosomiale

Spesso per le malattie lisosomiali l'unico trattamento è la terapia enzimatica sostitutiva, un'infusione endovenosa dell'enzima che il corpo non produce, cui il paziente deve sottoporsi per tutta la vita. I trattamenti, in media, richiedono dalle 2 alle 6 ore e vengono somministrati 1 volta ogni 7 giorni oppure ogni 14 giorni, in base al tipo di farmaco e alla patologia. Se si considera anche il tempo impiegato dai pazienti per raggiungere l'ospedale e il tempo di attesa presso la struttura in cui viene somministrata la terapia, ne derivano intere giornate di assenza dal lavoro o da scuola. Nel caso di paziente pediatrico con un genitore a sua volta malato, le assenze dovute alle terapie raddoppiano arrivando fino a 104 giorni l'anno, difficilmente giustificabili sul luogo di lavoro. Spesso, infatti (anche a causa del mancato riconoscimento di invalidità ai sensi della legge 104/1992) i pazienti sono costretti ad usufruire di permessi e ferie per poter effettuare le terapie.

A fronte di ciò, sono molti i vantaggi dalla terapia domiciliare (o home therapy), messi in evidenza da diversi studi condotti da clinici italiani, e ricordati nel corso del convegno del 24 ottobre 2018.

Per il servizio sanitario la terapia domiciliare presenta un enorme vantaggio economico, con l'azzeramento dei costi di day-hospital o day-service e la possibilità di disporre del personale infermieristico per altre funzioni o situazioni di emergenza.

Per il paziente crea la possibilità di concordare gli appuntamenti adattandoli alla sua quotidianità e quindi in orario extra-scolastico o extra-lavorativo; la riduzione delle assenze dal lavoro o da scuola; la riduzione del numero di viaggi per raggiungere l'ospedale e quindi una migliore gestione del tempo e risparmio dei costi di trasporto e parcheggio, ma anche la minore esposizione a rischio di incidenti stradali; infine, la regolarità nella frequenza della terapia in quanto non vincolata alla disponibilità di posti letto.

Inoltre, il paziente ha una minore percezione della malattia, migliorando così la propria qualità di vita anche a livello psicologico. Entrare in ospedale ogni settimana oppure ogni 14 giorni, costringe i pazienti ad adattare la loro vita alle terapie che gliela salvano. In molti Paesi europei e negli Stati Uniti la terapia domiciliare è ampiamente utilizzata, e in casi di malattie, come quella di Gaucher, è anche possibile l'auto-infusione, ulteriore modalità che potrebbe essere implementata nella nostra Nazione.

\section{Dichiarazione di assenza di conflitto di interessi}

Gli Autori dichiarano di non avere conflitti di interessi.

\section{Finanziamenti}

Gli Autori dichiarano di non aver ricevuto finanziamenti specifici da qualsiasi ente nei settori pubblico, privato o senza fini di lucro. 